\title{
3D Bone Mineral Density Distribution and Shape Reconstruction of the Proximal Femur from a Single Simulated DXA Image: An In Vitro Study
}

\author{
Tristan Whitmarsh ${ }^{a, b}$, Ludovic Humbert ${ }^{a, b}$, Mathieu De Craene ${ }^{b, a}$, Luís Miguel Del Rio \\ Barquero $^{c}$, Karl Fritscher ${ }^{e}$, Rainer Schubert ${ }^{e}$, Felix Eckstein ${ }^{f}$, Thomas Link ${ }^{g}$ and Alejandro F. \\ Frangi $a, b, d$ \\ ${ }^{a}$ Center for Computational Imaging \& Simulation Technologies in Biomedicine (CISTIB), \\ Universitat Pompeu Fabra, Barcelona, Spain; \\ ${ }^{b}$ Networking Research Center on Bioengineering, Biomaterials and Nanomedicine \\ (CIBER-BBN), Spain; \\ ${ }^{c}$ CETIR Centre Mèdic, Barcelona, Spain; \\ ${ }^{d}$ Institució Catalana de Recerca i Estudis Avançats (ICREA), Spain; \\ ${ }^{e}$ Institute for Biomedical Image Analysis, UMIT, Hall in Tirol, Austria; \\ Institute of Anatomy \& Musculoskeletal Research, PMU, Salzburg, Austria; \\ ${ }^{g}$ Musculoskeletal and Quantitative Imaging Research Group, UCSF, California, USA;
}

\begin{abstract}
Area Bone Mineral Density (aBMD) measured by Dual energy X-ray Absorptiometry (DXA) is an established criterion in the evaluation of hip fracture risk. The evaluation from these planar images however is limited to $2 \mathrm{D}$ while it has been shown that proper 3D assessment of both the shape and the BMD distribution improves the fracture risk estimation. In this work we present a method to reconstruct both the 3D bone shape and 3D Bone Mineral Density (BMD) distribution of the proximal femur from a single DXA image. A statistical model of shape and a separate statistical model of the BMD was automatically constructed from a set of quantitative computed tomography (QCT) scans. The reconstruction method incorporates a fully automatic intensity based 3D-2D registration process, maximizing the similarity between the DXA and a digitally reconstructed radiograph of the combined model. For the experiments an in vitro dataset of QCT scans of 90 anatomical specimens was used. Out of these, 60 were used to construct both the shape and the density model. To evaluate the reconstruction accuracy, experiments were performed on simulated DXA images from the remaining 30 QCT scans. Comparisons between the reconstructions from DXA with the same subject QCT scans showed a mean shape accuracy of $1.2 \mathrm{~mm}$ whereby $95 \%$ of the error is below $3.2 \mathrm{~mm}$, and a mean density error of $81 \mathrm{mg} / \mathrm{cm}^{3}$ corresponding to $4.6 \%$ of the whole range of bone density values. The results show that this method is capable of accurately reconstructing both the 3D shape and 3D BMD distribution of the proximal femur from DXA images used in clinical routine, potentially improving the diagnosis of osteoporosis and fracture risk assessments at a low radiation dose and low cost.
\end{abstract}

Keywords: Statistical Model, Registration, Reconstruction, Osteoporosis

\section{INTRODUCTION}

Femur fracture due to osteoporosis affects about $18 \%$ of women over 50 years old and accounts for significant morbidity, disability, decreased quality of life, mortality and high economic cost for society. ${ }^{1}$ This indicates the importance of an accurate diagnosis of osteoporosis and fracture risk assessment. Osteoporosis diagnosis is currently performed by computing from Dual-energy X-ray Absorptiometry (DXA) a Bone Mineral Density

Further author information: (Send correspondence to T. Whitmarsh)

T. Whitmarsh: E-mail: twhitmarsh@cistib.upf.edu, Telephone:

L. Humbert: E-mail: lhumbert@cistib.upf.edu, Telephone: 
(BMD) index related to the total bone mass. Although DXA gives an accurate planar representation of the BMD, it is limited by its two-dimensionality and does not represent the shape or spatial distribution of the bone mass. These have been shown to be essential parameters for an accurate fracture risk prediction. ${ }^{2}$ A $3 \mathrm{D}$ reconstruction of the femur bone with bone mass distribution can be acquired by quantitative computed tomography (QCT). Here a calibration phantom is used to relate the Hounsfield number to a volumetric bone density $\left(\mathrm{g} / \mathrm{cm}^{3}\right)$. This medical imaging technique, however, is associated with high financial costs, high radiation doses for the patient and is not used in clinical routine for osteoporosis follow-up.

3D reconstruction methods from commonly-used DXA images have not been investigated much. Langton et $a .^{3}{ }^{3}$ proposed a 3D reconstruction method from a single DXA image by deforming a generic model. This method, however, is limited to the 3D reconstruction of the shape only. By using a statistical model describing the 3D shape (and BMD distribution) of a population, a probabilistic reconstruction of the shape (and BMD distribution) can be made, overcoming the 2D limitations of a single DXA image. In Fritscher et al. ${ }^{4}$ a fully automatic approach to building a statistical shape-intensity model was presented. It has however not been used to reconstruct the shape and density distribution from DXA. In Yao and Taylor ${ }^{5}$ a method for the construction of a statistical bone density atlas is described whereby a tetrahedral mesh structure is equipped with embedded Bernstein polynomial density functions on the barycentric coordinates of the tetrahedrons. In Ahmad et al. ${ }^{6}$ this tetrahedral model was registered onto two or more DXA images. The model, however, does not describe the variation in BMD distribution over a population. Therefore no patient-specific reconstruction of the BMD distribution can be acquired through the registration of this model. In addition, the presented experiments rely on multiple views generated by a C-arm DXA device. These devices are still not widely used in clinical routine. We therefore propose a method to reconstruct the proximal femur from a single DXA image acquired from single view densitometry devices, which are the most commonly used devices in clinical practice.

This study presents and evaluates a method to fully automatically reconstruct both the 3D BMD distribution and shape of the proximal femur from a single 2D DXA image. Our reconstruction method incorporates a statistical model of the shape together with a statistical model of the density, built from a database of 3D QCTscans, which encodes the variations of shape and BMD distribution in the population. A 3D-2D registration technique was developed to find an instance of both models so that the projection of the density model, deformed according to the shape model, corresponds with the 2D DXA image. Finally, the accuracy of the method was established by in vitro experiments.

\section{METHODOLOGY}

We first developed a method to automatically build a 3D statistical deformable model of shape from QCT-scan acquisitions based on previous research. ${ }^{7}$ Next a statistical density model was built from the BMD information in the QCT volumes. Our reconstruction method follows the principle of Active Appearance Models (AAM) as introduced by Cootes et al. ${ }^{8}$ whereby the density model is deformed according to the shape model. This combined model was finally registered onto the DXA images resulting in patient specific reconstructions.

\subsection{Shape model construction}

To built the shape model, first a reference QCT volume is chosen manually, based on its regular bone shape. All volumes are registered onto this reference volume by means of an affine transformation followed by a multi scale B-spline registration. For the multi scale B-spline registration we used a control point spacing of 32, 16 and $8 \mathrm{~mm}$ consecutively whereby the displacement of the control points are constrained to 0.4 times the control point spacing to guarantee diffeomorphism. ${ }^{9}$ The reference volume is then segmented using ITK-SNAP. ${ }^{10}$ This software provides a semi-automatic segmentation using active contour methods. Of this segmentation a detailed surface mesh is constructed. For each volume both the affine and B-spline transformations are then applied to this surface mesh. This results in a surface mesh of the bone for each subject. Each surface mesh is then normalized so that the average distance of the surface points to the center of mass is scaled to a constant. Generalized Procrustes Analysis (GPA) was then used to align the normalized surface meshes. Each $i$-th shape can now be represented by a shape vector $\mathbf{s}_{i}=\left(x_{1}, y_{1}, z_{1}, \ldots, x_{n}, y_{n}, z_{n}\right)^{T} \in \mathbb{R}^{3 n}$ defined by the $n$ vertices. Principal component analysis (PCA) is then applied to the data matrix $\mathbf{D}_{\text {shape }}=\left[\left(\mathbf{s}_{1}-\overline{\mathbf{s}}\right)\left|\left(\mathbf{s}_{2}-\overline{\mathbf{s}}\right)\right| \cdots \mid\left(\mathbf{s}_{k}-\overline{\mathbf{s}}\right)\right]$ where $k$ is the number of shapes and $\overline{\mathbf{s}}$ the average shape. PCA computes an orthonormal basis aligning the axes with 
the maximum variation in the data. The axes are given by the ordered eigenvectors of the covariance matrix of $\mathbf{D}_{\text {shape }}$ which are also referred to as the modes of variation. Now a new shape can be represented as the average shape plus the first $p$ eigenvectors (Figure 1) whereby each eigenvector $\mathbf{e}_{a}$ is multiplied by a corresponding scalar weight $\alpha_{a}$ :

$$
\mathbf{s}_{\text {mod }}=\overline{\mathbf{s}}+\sum_{a=1}^{p} \alpha_{a} \mathbf{e}_{a}
$$

\subsection{Density model construction}

To capture only the density variations irrespective of the shape, the previously acquired segmentations are used to deform each volume to the mean of the shape model using a Thin Plate Spline (TPS) transformation. ${ }^{11}$ By using only the points on the bone surface for the TPS transformation, the spatial distribution of the BMD inside the bone will be preserved. The resulting volumes now have the same average bone shape with a voxel correspondence between them. The voxel densities in each $j$-th volume can now be represented as a vector $\mathbf{v}_{j}=\left(d_{1}, d_{2}, \ldots, d_{m}\right)^{T} \in \mathbb{R}^{m}$ where $m$ is the number of voxels inside the shape and $d_{m}$ the density of the $m$-th voxel. PCA is then applied to the data matrix $\mathbf{D}_{\text {densities }}=\left[\left(\mathbf{v}_{1}-\overline{\mathbf{v}}\right)\left|\left(\mathbf{v}_{2}-\overline{\mathbf{v}}\right)\right| \cdots \mid\left(\mathbf{v}_{l}-\overline{\mathbf{v}}\right)\right]$ where $l$ is the number of volumes and $\overline{\mathbf{v}}$ the average volume. A new volume can now be represented as the average volume plus the first $q$ eigenvectors (Figure 2), whereby each eigenvector $\mathbf{f}_{b}$ is multiplied by a corresponding scalar weight $\beta_{b}$ :

$$
\mathbf{v}_{\text {mod }}=\overline{\mathbf{v}}+\sum_{b=1}^{q} \beta_{b} \mathbf{f}_{b}
$$

Horn's parallel analysis ${ }^{12}$ was used to determine the required number of modes. Using the dataset described in Section 3 this resulted in 14 modes for the shape model and 11 modes for the density model (Figure 3).

\subsection{Reconstruction}

Our reconstruction method is based on the registration of the combined shape and density model onto a DXA image. This is achieved by finding the instance of the shape model and the instance of the density model, together with a similarity transform (translation, rotation and uniform scaling), that maximizes the similarity between DXA and the Digitally Reconstructed Radiograph (DRR) generated from the combined model.

Similar to Cootes et al. ${ }^{8}$ the shape model generates a new shape onto which an instance of the density model is deformed using a TPS transformation. For this transformation, the vertices of the average shape $\overline{\mathbf{s}}$ represent the source points while the target points are defined by the vertices of the new shape model instance $\mathbf{s}_{\text {mod }}$.

In an intensity based registration process, the parameter space, representing the shape and density model parameters and similarity transform, is searched to maximize the similarity between the DXA and DRR of the deformed density model instance. To find the parameters that maximizes this similarity, defined in terms of the mean absolute error, Powell's multidimensional optimization method ${ }^{13}$ is used.

The DRR is generated using a ray casting technique ${ }^{14}$ resulting in a $2 \mathrm{D}$ image representing the aBMD $\left(\mathrm{g} / \mathrm{cm}^{2}\right)$. For each position $\gamma$ in the DRR image, a set of sample points $\mathbf{R}_{\gamma}$ is taken at regular intervals along the ray. Each sample point is transformed by the similarity transform $\mathbf{T}_{s}$ and the TPS transformation $\mathbf{T}_{t p s}$ consecutively. The density at the resulting position is then taken from the density model instance volume $\mathbf{V}_{\text {mod }}$ which is reconstructed from the density model instance vector $\mathbf{v}_{\text {mod }}$ (Equation 1). The aBMD at position $\gamma$ of the DRR can thus be formulated as:

$$
\operatorname{DRR}(\gamma)=\sum_{r \in \mathbf{R}_{\gamma}} \mathbf{V}_{\text {mod }}\left(\mathbf{T}_{t p s}\left(\mathbf{T}_{s}(r)\right)\right) \delta
$$

where $\delta$ is the distance between the sample points. 


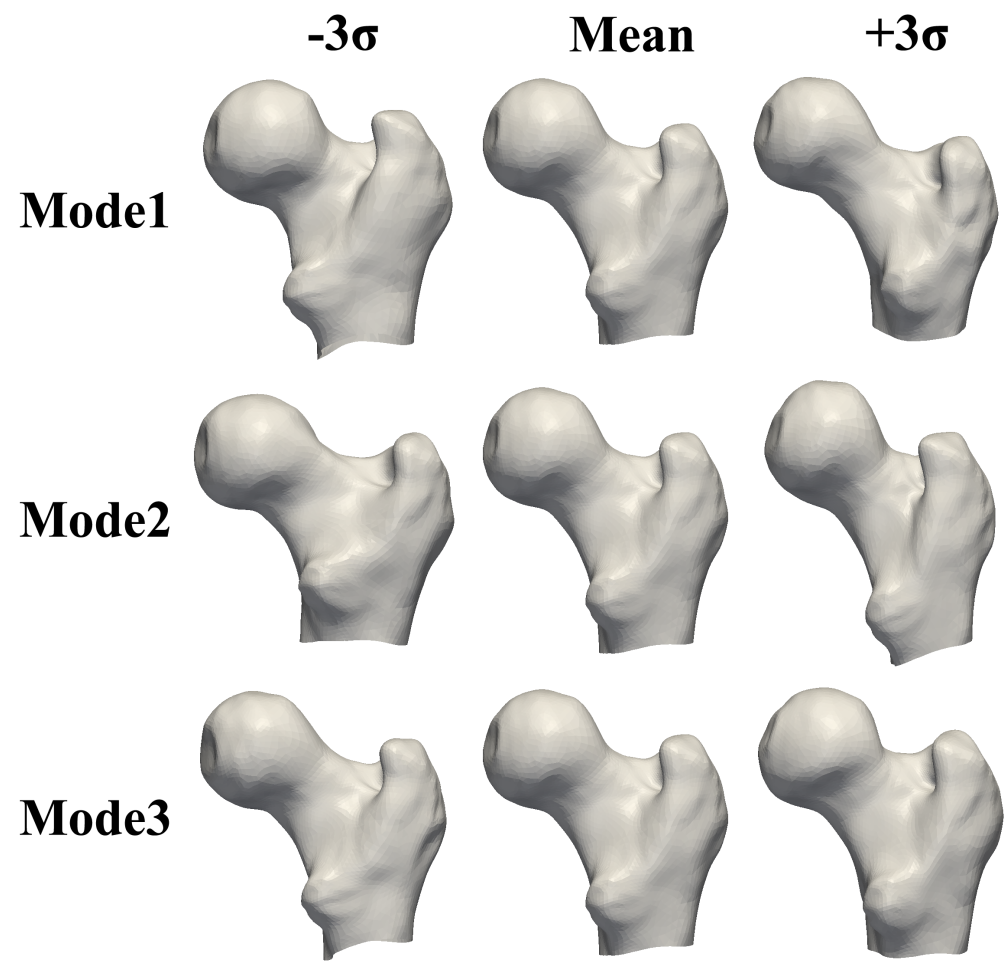

Figure 1. The mean and the first three modes of variation of the geometric model, varying between -3 and +3 standard deviations $(\sigma)$ obtained from the database described in Section 3.

\section{METHOD EVALUATION}

A database of 90 specimens of human proximal femurs, collected for a previous study ${ }^{15}$ from the Institute of Anatomy at the Ludwig Maximilians University Munich (Germany) was used. All these bones were scanned using a 16-row MSCT scanner (Sensation 16; Siemens Medical Solutions, Erlangen, Germany). All the CT-scans were resampled from a spatial resolution of $0.195 \times 0.195 \times 0.5 \mathrm{~mm}^{3}$ to a spatial resolution of $0.5 \times 0.5 \times 0.5 \mathrm{~mm}^{3}$ and calibrated using a phantom to relate the Hounsfield value to a BMD. Of this database of volumes, 30 were taken for the validation of the reconstruction method. Of those 30 subjects with a mean age of $77 \pm 11$ years, 15 were male and 15 female whereby for both, 5 subjects were defined as osteoporotic, 5 as osteopenic and 5 were considered healthy based on the WHO criteria (BMD measurements had been performed from frontal DXA images of the specimens, however, these DXA images have not been stored). Of the remaining 60 volumes, consisting of 28 men and 32 woman with a mean age of $79 \pm 11$ years, the shape and density model was constructed.

Since the DXA image acquisitions of these specimens were not available, simulated DXA images were generated from the QCT volumes in the same manner as for the DRR generation (Section 2.3). For the simulated DXA images we assume a rectilinear narrow fan-beam DXA device similar to GE Healthcare's Lunar iDXA (GE Healthcare, Chalfont St. Giles, UK). In such device a post processing reconstruction method eliminates the inherent magnification and distortion effects of the fan-beam projection. As a result the projection can be represented a an isometric projection. The simulated DXA images were given a resolution of $0.3 \times 0.3 \mathrm{~mm}^{2}$ corresponding to the Lunar iDXA output.

The QCT volumes used for generating the simulated DXA images served as the "ground truth" volumes. To evaluate the shape reconstruction accuracy, we segmented every bone in the validation dataset (again using ITK-SNAP) and for each segmentation constructed the surface mesh. These were then considered the "ground truth" for the individual shapes. Each mesh resulting from the reconstruction was registered onto its corresponding "ground truth" mesh by an Iterative Closest Point (ICP) registration. We then get the reconstruction 


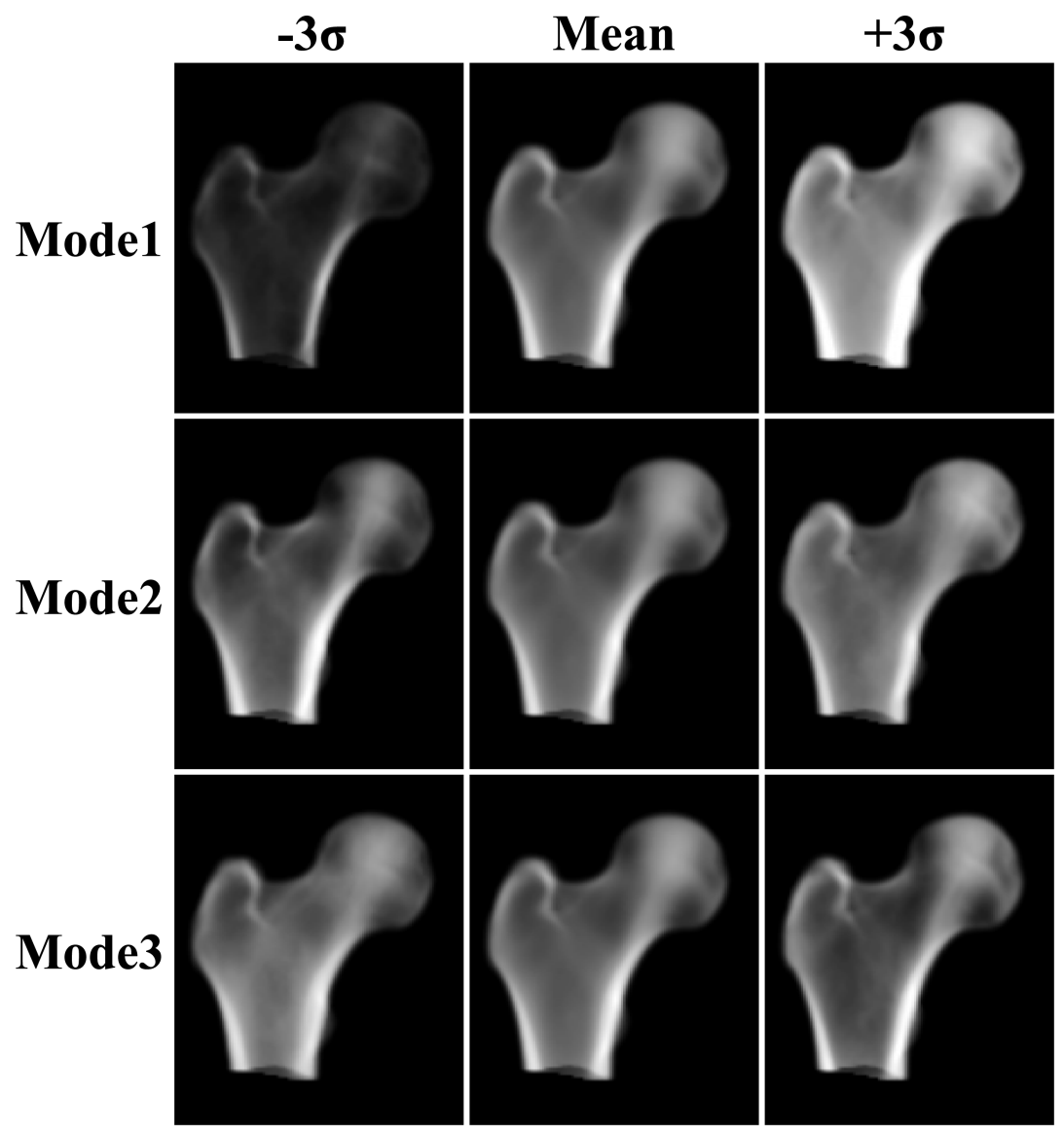

Figure 2. Projections of the mean and the first three modes of variation of the density model, varying between -3 and +3 standard deviations $(\sigma)$ obtained from the database described in Section 3.

error by finding the point-to-surface distances of every vertex in the reconstructed shape mesh to the "ground truth" surface mesh. To evaluate the reconstruction accuracy of the BMD distribution, we first apply the transformation resulting from the previous ICP registration to the reconstructed volume to align them with their corresponding "ground truth" volume. The density difference between the voxels of the reconstructed volume and the corresponding voxels in the "ground truth" volume was then taken as the BMD distribution error.

\section{RESULTS AND DISCUSSION}

For the shape accuracy we obtained an average error of $1.2 \mathrm{~mm}$ and a 2 Root Mean Squared (2RMS) error of $3.2 \mathrm{~mm}$, giving a $95 \%$ confidence interval for the shape accuracy. In Kolta et al. ${ }^{16}$ a mean point to surface distance of $0.8 \mathrm{~mm}$ and a $2 \mathrm{RMS}$ error of $2.1 \mathrm{~mm}$ was reported. This however resulted from a reconstruction from two DXA images (frontal and sagittal) whereas our reconstruction method requires only one DXA image. In addition this method is limited to the shape reconstruction only. In Figure 5 an error map illustrates the point to surface distances averaged over all 30 subjects for every vertex on the shape model. Analyzing the reconstruction accuracy of the BMD distribution, a mean absolute error of $81 \mathrm{mg} / \mathrm{cm}^{3}$ corresponding to $4.6 \%$ of the average range of bone densities $\left(1776,69 \mathrm{mg} / \mathrm{cm}^{3}\right)$ was measured with a $95 \%$ confidence interval of $285 \mathrm{mg} / \mathrm{cm}^{3}$ corresponding to $16 \%$ of the average range of bone densities. A volume reconstruction accuracy is given in Fritscher et al. ${ }^{17}$ whereby the error corresponds to $2.8 \%$ of the whole intensity range. This however was result of a registration of the model onto a volume as opposed to our reconstruction method from a single projection. Some results of the reconstructions of various shapes and BMD distributions are shown in Figure 4 illustrating the large variation in shape and BMD distribution the models can capture. 

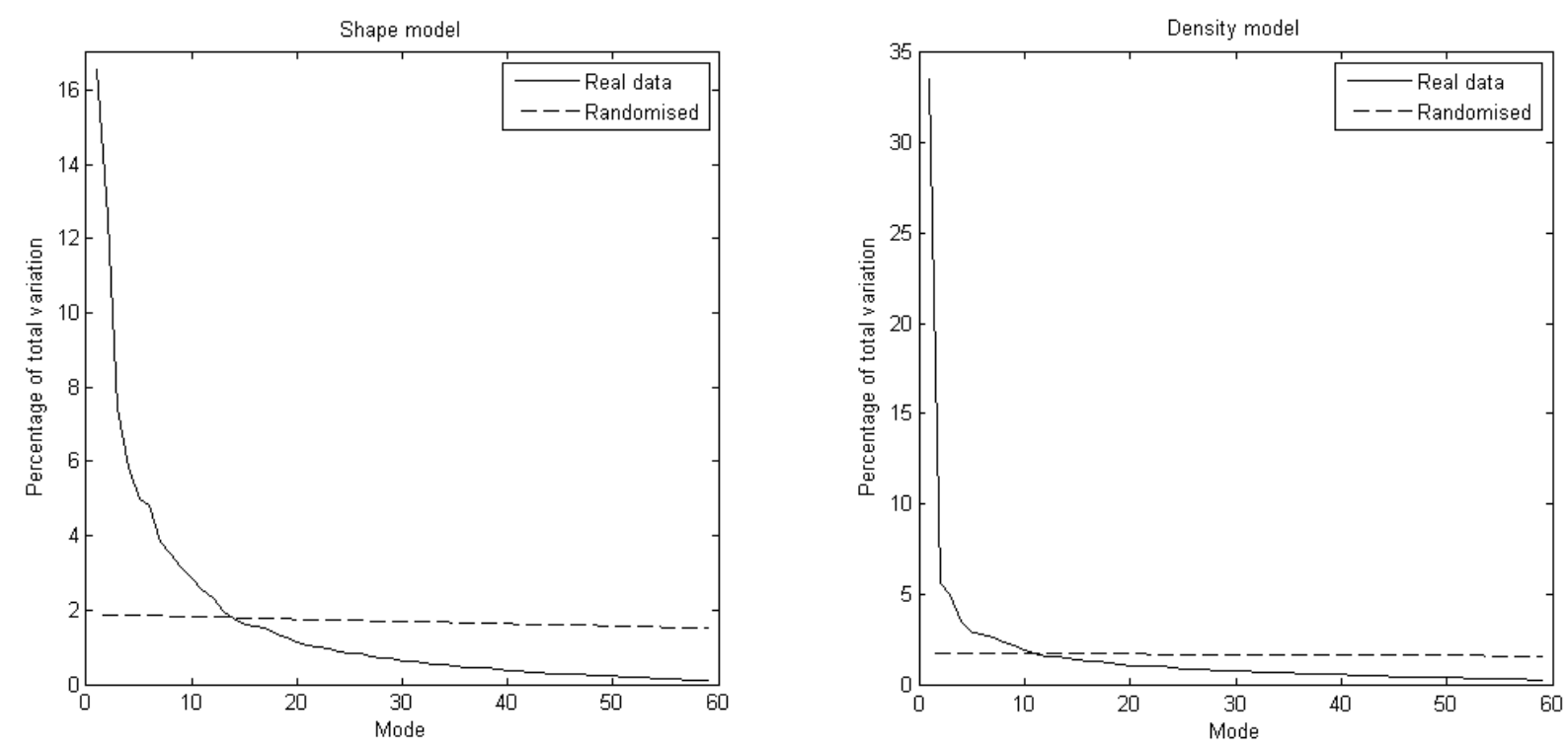

Figure 3. A plot of the eigenvalues set against the mode, compared to a randomized version of the data for both the shape model (left) and the density model (right). The plots intersect approximately at mode 11 for the shape model and at mode 14 for the density model.

It is possible to combine the geometry and densities in a single model thereby capturing the relation between the shape and the BMD distribution as proposed in Schuler et al. ${ }^{18}$ and originally proposed by Cootes et al. ${ }^{8}$ We however propose to build a separate model for the densities and shape thereby decoupling the BMD density information from the shape. This is important for osteoporosis follow-up whereby the BMD distribution change is examined irrespective of the shape. Since the bone mineral density and geometry of the proximal femur differs significantly between men and woman, ${ }^{19}$ it would be beneficial to construct a separate model for each gender as in Ahmad et al. ${ }^{6}$ We however do not posses a large enough dataset to be able to acquire meaningful statistics on the reconstruction accuracy of male and females separately and we therefore opted to combine male and female subjects into a single model. Because the shape model is built from a dense surface mesh, a subset of the vertices are taken for the TPS transformations. This fortuitously allows for a coarse to fine registration allowing a more coarse model to be registered initially, followed by a more detailed model, decreasing the computation time and possibly preventing the convergence to a local minimum.

\section{CONCLUSION}

To the best of our knowledge this is the first attempt to reconstruct both the shape and the 3D BMD distribution from a single DXA image. Our study is based on a statistical model of shape together with a statistical model of BMD, built from QCT-scan volumes. By incorporating statistics of a large population, a 3D reconstruction from a single DXA image can be obtained. Additionally, the use of an image based registration process together with our density model aids in the 3D shape estimation by making use of all the information available in the $2 \mathrm{D}$ radiograph as opposed to landmark based registration methods. ${ }^{16}$ Although methods based on conventional X-rays allow the 3D reconstruction of shape, DXA images allows us to reconstruct the shape as well as the 3D BMD distribution of the bone. Comparisons between the reconstructed femur and "ground truth" show that our method results in accurate and realistic reconstructions.

Our future work aims at improving the diagnosis of osteoporosis and fracture risk assessment from DXA by using accurate information of the 3D shape and BMD distribution obtained by the presented DXA-based reconstruction method. We aim to demonstrate that the advantages of a 3D analysis on fracture risk assessment accuracy can be implemented in clinical practice, maintaining DXA as the current standard modality. For this, our reconstruction method will be validated on in-vivo data and real DXA images. 

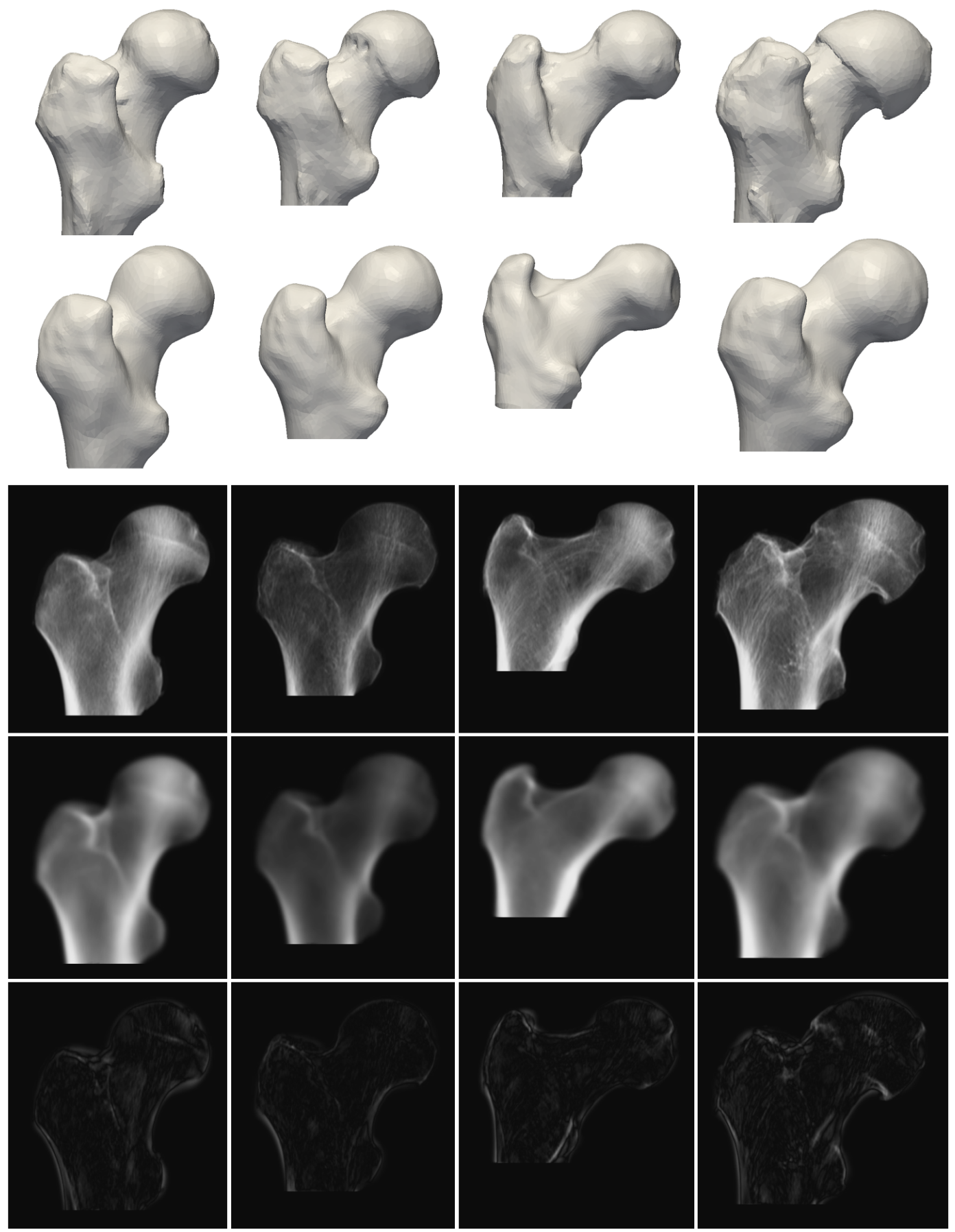

Figure 4. Results of four of the reconstructions. From top to bottom: the "ground truth" surface mesh, the reconstructed shape, the simulated DXA image, the projections of the model resulting from the registrations and the difference images (image of the absolute values of the subtraction between the projection of the model and the DXA image). 

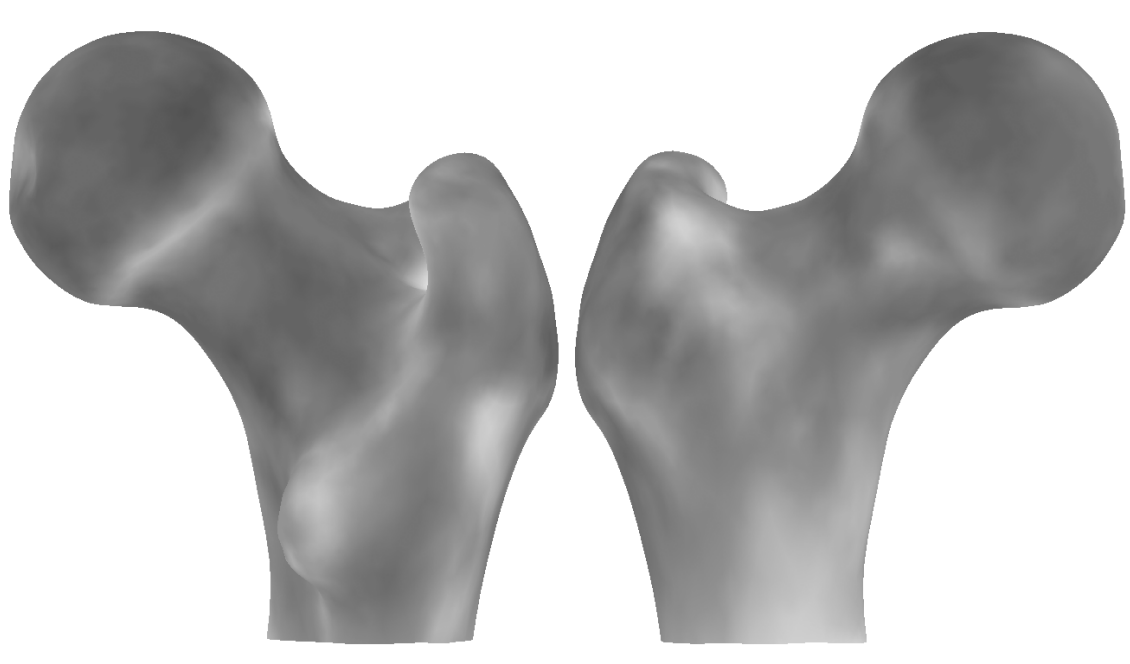

error (mm)

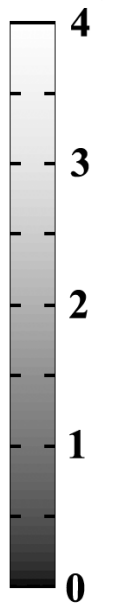

Figure 5. The color-coded point to surface distances $(\mathrm{mm})$ between the reconstructed shapes and the QCT-derived surfaces, averaged over the 30 subjects.

In this paper we proposed a 3D reconstruction method of the shape and BMD distribution from a single DXA image by using the intensity-based registration of a combined shape and density statistical model. This method is compliant with clinical routine since DXA scanners are mostly limited to the acquisition of a single view. This opens the way for a better diagnosis of osteoporosis, by providing a detailed 3D analysis of the femur from routine low cost and low radiation dose 2D DXA imaging devices.

\section{ACKNOWLEDGMENTS}

This research has been financially supported by the 3D-FemOs project (3-Dimensional Femur Reconstruction for Osteoporotic Fracture Risk Assessment) with a grant awarded by ACC1Ó Valtec (Tipus 1). In addition this work has been supported by a grant from the Instituto de Salud Carlos III (FI09/00757) and by a grant from the "Deutsche Forschungsgemeinschaft" (LO 730 / 3-1). The authors acknowledge Benedikt Schuler (Institute for Biomedical Image Analysis, UMIT, Hall in Tirol, Austria) and Dr. E. M. Lochmüller (Universitäts-Frauenklinik der LMU, Mnchen, Germany) for the data collection.

\section{REFERENCES}

[1] WHO, [Prevention and management of osteoporosis], World Health Organization Technical Report Series 921 (2003).

[2] Bras, A. L., Kolta, S., Soubrane, P., Skalli, W., Roux, C., and Mitton, D., "Assessment of femoral neck strength by 3-dimensional x-ray absorptiometry," Journal of Clinical Densitometry 9(4), 425 - 430 (2006).

[3] Langton, C. M., Pisharody, S., and Keyak, J. H., "Generation of a 3d proximal femur shape from a single projection 2d radiographic image," Osteoporosis International 20, 455-461 (Mar. 2009).

[4] Fritscher, K., Grünerbl, A., and Schubert, R., "3d image segmentation using combined shape-intensity prior models," International Journal of Computer Assisted Radiology and Surgery 1, 341-350 (Apr. 2007).

[5] Yao, J. and Taylor, R., "Assessing accuracy factors in deformable 2d/3d medical image registration using a statistical pelvis model," Computer Vision, 2003. Proceedings. Ninth IEEE International Conference on , 1329-1334 vol.2 (Oct. 2003).

[6] Ahmad, O. M., Ramamurthi, K., Wilson, K. E., Engelke, K., Bouxsein, M., and Taylor, R. H., "3d structural measurements of the proximal femur from 2d dxa images using a statistical atlas," Medical Imaging 2009: Computer-Aided Diagnosis 7260(1), 726005, SPIE (2009). 
[7] Frangi, A., Rueckert, D., Schnabel, J., and Niessen, W., "Automatic construction of multiple-object threedimensional statistical shape models: application to cardiac modeling," IEEE Transactions on Medical Imaging 21(9), 1151-1166 (2002).

[8] Cootes, T. F., Edwards, G. J., and Taylor, C. J., "Active appearance models," IEEE PAMI 23(6), 681-685 (2001).

[9] Rueckert, D., Aljabar, P., Heckemann, R., Hajnal, J., and Hammers, A., "Diffeomorphic registration using b-splines," Lecture Notes in Computer Science 4191, 702 (2006).

[10] Yushkevich, P. A., Piven, J., Hazlett, H. C., Smith, R. G., Ho, S., Gee, J. C., and Gerig, G., "User-guided 3d active contour segmentation of anatomical structures: Significantly improved efficiency and reliability," NeuroImage 31(3), 1116 - 1128 (2006).

[11] Bookstein, F., "Principal warps: Thin-plate splines and the decomposition of deformations," IEEE Transactions on Pattern Analysis and Machine Intelligence 11(6), 567-585 (1989).

[12] Horn, J., "A rationale and test for the number of factors in factor analysis," Psychometrika 30, 179-185 (June 1965).

[13] Press, W., Flannery, B., Teukolsky, S., and Vetterling, W., [Numerical Recipes in C: The Art of Scientific Computing], Cambridge University Press (Oct. 1992).

[14] Burghardt, A., Kazakia, G., Link, T., and Majumdar, S., "Automated simulation of areal bone mineral density assessment in the distal radius from high-resolution peripheral quantitative computed tomography," Osteoporosis International 20, 2017-2024 (Dec. 2009).

[15] Eckstein, F., Wunderer, C., Boehm, H., Kuhn, V., Priemel, M., Link, T. M., and Lochm??ller, E.-M., "Reproducibility and side differences of mechanical tests for determining the structural strength of the proximal femur," Journal of Bone and Mineral Research 19(3), 379-385 (2004). PMID: 15040825.

[16] Kolta, S., Le Bras, A., Mitton, D., Bousson, V., De Guise, J. A., Fechtenbaum, J., Laredo, J. D., Roux, C., and Skalli, W., "Three-dimensional x-ray absorptiometry (3d-xa): A method for reconstruction of human bones using a dual x-ray absorptiometry device," Osteoporosis Int. 16(8), 969-976 (2005).

[17] Fritscher, K., Schuler, B., Link, T., Eckstein, F., Suhm, N., Hänni, M., Hengg, C., and Schubert, R., "Prediction of biomechanical parameters of the proximal femur using statistical appearance models and support vector regression," in [MICCAI '08: Proceedings of the 11th international conference on Medical Image Computing and Computer-Assisted Intervention - Part I], 568-575, Springer-Verlag, Berlin, Heidelberg (2008).

[18] Schuler, B., Fritscher, K. D., Kuhn, V., Eckstein, F., and Schubert, R., "Using a statistical appearance model to predict the fracture load of the proximal femur," Medical Imaging 2009: Visualization, Image-Guided Procedures, and Modeling 7261(1), 72610W, SPIE (2009).

[19] Tuck, S. P., Pearce, M. S., Rawlings, D. J., Birrell, F. N., Parker, L., and Francis, R. M., "Differences in bone mineral density and geometry in men and women: the newcastle thousand families study at 50 years old," Br J Radiol 78(930), 493-498 (2005). 